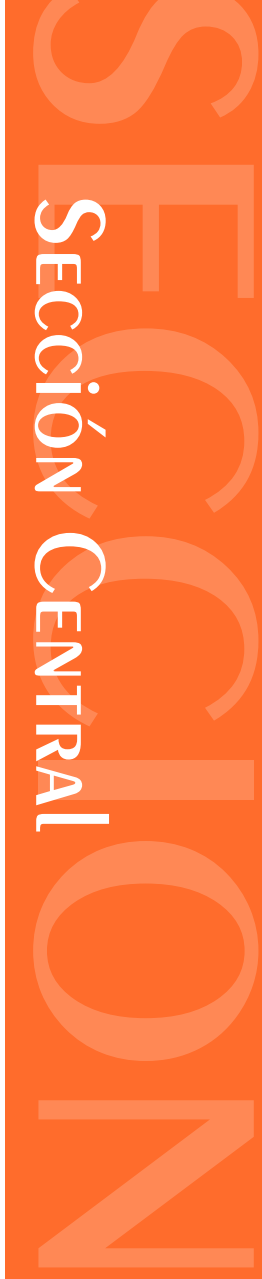




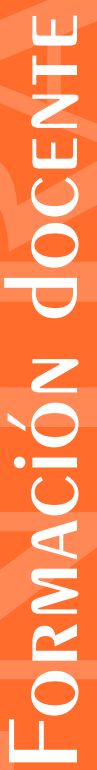
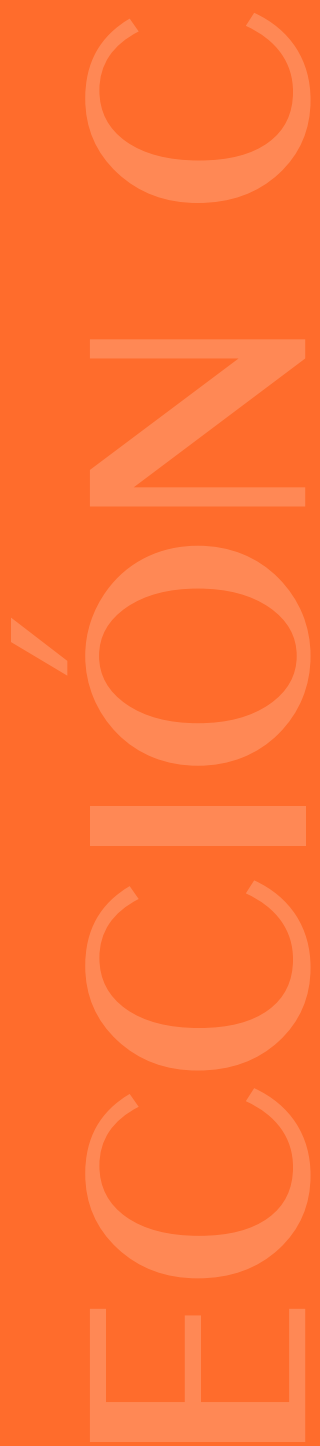


\section{INICIOS Y PRESENTE DE LA FORMACIÓN DE DOCENTES PARA LA EDUCACIÓN FÍSICA COLOMBIANA}

ORIGINS AND PRESENT FOR TEACHER TRAINING IN COLOMBIAN PHYSICAL EDUCATION

Germán Preciado Mora ${ }^{1}$

\section{Resumen}

Este artículo rinde un merecido homenaje a la Educación Física colombiana, que el 25 de junio de 2016 celebró con grandes pompas su octogésimo aniversario de acción pedagógica profesional en Colombia. En él, se describen los orígenes de la formación de educadores para el área y se avanza en un recorrido histórico por los principales hechos que han contribuido al presente de esta formación, la cual, al año 2016, cuenta con una amplia gama de posibilidades y ofertas dentro de la estructura de nuestro sistema educativo. Así mismo, cuenta con un sinnúmero de titulaciones, de posibilidades de ejercicio profesional, y de actualización y formación posgradual que la ubican como una de las mejores alternativas profesionales para los jóvenes que buscan delinear un proyecto de vida personal y profesional en Colombia.

Palabras clave: formación de docentes, docentes de Educación Física, formación de educadores físicos, orígenes de la formación de educadores físicos

\section{Abstract}

This article pays tribute to Colombian Physical Education, which on June 25th 2016, celebrated with great pomp its eightieth anniversary of professional pedagogical action in Colombia. In this article, the origins of teacher training for the area are described and, at the same time, it is made a historical itinerary for the major events that have contributed to the present of this training. At this time, 2016, it has a wide range of possibilities and offerings, within the structure of our educational system. In addition, it has a number of qualifications, opportunities for practice, and training posgraduate updates that place it as one of the best alternatives for young professionals looking for a personal and professional life in Colombia.

Keywords: teacher training, Physical education teachers, physical educator training, origins of physical educators training

Fecha de recepción: 4 de diciembre de 2015

Fecha de aprobación: 4 de mayo de 2016

Para citar este artículo:

Preciado, G. (2016). Inicios y presente de la formación de docentes para la educación física colombiana. Lúdica Pedagógica, 24,

$11-22$.

1 Licenciado en Educación Física, Universidad Pedagógica Nacional. Magíster en Administración, Universidad de la Salle. Docente investigador de la Secretaría de Educación de Bogotá. Gerente de Gerpremo Inversiones. Leader Coach del Nodo Actividad Física y Cuerpo de la Red Distrital de Docentes Investigadores, REDDI, y de la RED Local de Educación Física de Kennedy. Promotor y practicante activo de la Educación Física Mental y del Walking Life. Correo electrónico: gerpremo@yahoo.es - gerpremo@gmail.com 
Figura 1. Clase de Tenis de campo para docentes en formación en la Universidad Pedagógica Nacional.

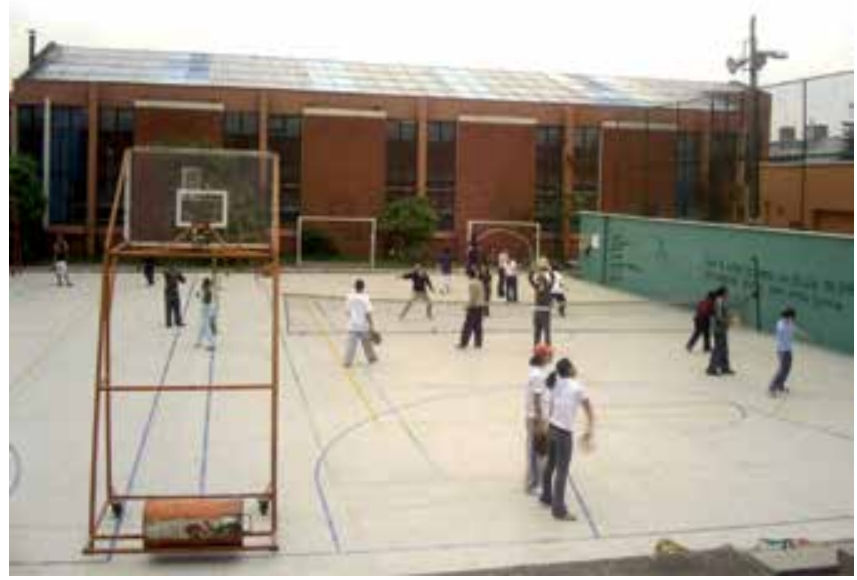

Fuente: Mg. Germán Preciado Mora

\section{INTRODUCCIÓN}

El ser humano encuentra en el festejo y en la celebración de las efemérides una oportunidad para recordar, conmemorar o agradecer aquello que le da sentido a su existencia, de la mano con las personas que conforman la organización a la que -por decisión, costumbre, o tradición- pertenece, sea esta su familia o la comunidad con la que habita, trabaja o estudia. Este año en particular, la comunidad educativa colombiana celebra el octogésimo aniversario de acción pedagógica de la Educación Física como profesión, y una forma de conmemorarlo es traer al presente los sucesos que han hecho posible su presencia tanto en la educación superior (como posibilidad para el desarrollo personal y profesional de los jóvenes colombianos) como en la escuela, contribuyendo al desarrollo integral y armónico de los niños, niñas y jóvenes del país; y finalmente en la sociedad, fomentando la promoción de la salud, la calidad de vida y el bienestar físico de millones de colombianos a lo largo de nuestra extensa y variada geografía.

El presente artículo pretende mostrar varios de esos sucesos y realizar un recorrido histórico que permita entender el pasado, reconocer el presente y orientar la proyección del futuro de esta disciplina pedagógica y actividad física planeada, la cual inició en la ciudad de Bogotá, un jueves 25 de junio de 1936 y hoy, 80 años después, está presente como profesión en 36 municipios y ciudades de Colombia, en 48 Instituciones de Edu- cación Superior y 137 Escuelas Normales Superiores, que ofertan un total de 84 programas de formación a la comunidad.

\section{INICIOS DE LA FORMACIÓN DE DOCENTES PARA LA EDUCACIÓN FÍSICA COLOMBIANA}

La primera referencia a formación profesional de docentes para el área de Educación Física en Colombia se hace el miércoles 18 de noviembre de 1925, en el artículo 6 de la Ley 80 (1925), al indicar las atribuciones del técnico miembro obligatorio de la Comisión Nacional de Educación Física, ordenando: “a) Dar cursos intensivos para la preparación de maestros que han de poner al frente de las plazas de deportes." (p. 1). Luego, en el artículo 14 dispone: "El Ejecutivo podrá contratar en el país o en el exterior un técnico que inicie los cursos intensivos para la preparación de los maestros y que sea el Jefe de la Sección de Educación Física Nacional" (p. 1). El primer técnico nombrado por el gobierno del presidente Miguel Abadía Méndez en cumplimiento de la mencionada Ley fue el señor Hans Huber, miembro de la segunda Misión Pedagógica Alemana, quien en el año 1927 dirigió los dos primeros cursos informativos para maestros de primer y segundo grado de enseñanza. Estos cursos se trabajaban los días lunes y miércoles, de 4 a 5 p.m. el curso primero y de 5 a 6 p.m., el segundo. A ellos asistieron en total 45 maestros, 21 en el primer curso y 24 en el segundo. En el parágrafo de la Resolución No. 52, del 10 de mayo de 1927, se especifica que los cursos tendrán el siguiente plan de trabajo:

CURSO PRIMERO: Para maestros de primera enseñanza, que trabajarán divididos en tres grupos según la edad, los siguientes contenidos: Ejercicios libres de gimnasia sueca y alemana, Ejercicios con aparatos, Juegos, Carreras, Saltos y Lanzamientos y el CURSO SEGUNDO: Para maestros de Colegios de segunda enseñanza, que trabajarán los mismos contenidos del primer curso, pero con ejercicios propios para la edad de los alumnos (p. 1).

La terminación del contrato del profesor Hans Huber en 1929, la crisis económica de los años 30 y la guerra con el Perú, influyeron en la continuidad de este tipo de formación y en el desarrollo de la Educación Física, que se estancó incluso hasta el punto de desintegrarse la Comisión Nacional de Educación Física. 
El jueves 19 de octubre de 1933, el Gobierno del presidente Enrique Olaya Herrera expide el Decreto 1734 reglamentario de la Ley 80 de 1925, tratando de restaurar la Sección de Educación Física y estableciendo en el artículo 20 que "Tan pronto como las necesidades de los deportes lo justifiquen, la Comisión Nacional de Educación Física creará escuelas de Educación Física" (p. 3). Dicha Comisión determina que existe en el territorio nacional una falta de profesores de Educación Física que puedan dar cabal cumplimiento al Decreto mencionado y al Decreto 1792 de noviembre 1 de 1933, el cual estableció, por petición de algunos directores de Colegios de segunda enseñanza, que, a partir del año 1934, todos los aspirantes a obtener certificados de cultura general e ingreso a la universidad deberían haber cursado Educación Física; para el caso de las mujeres 2 horas semanales, de primero a sexto año, y para los varones, 3 horas de primero a tercer año y 2 horas de cuarto a sexto. Como medida de emergencia, se decide enviar en el año 1934 a un grupo de estudiantes a Santiago de Chile, para que reciban una instrucción especial en el Instituto Superior de Educación Física de la Universidad de Chile, entidad dirigida por el ilustre maestro Joaquín Cabezas García y con una amplia trayectoria y reconocimiento mundial en la formación de maestros para la Educación Física.

A través de la Comisión Nacional de Educación Física, y aprovechando los contactos establecidos con el Instituto Superior de Educación Física de la Universidad de Chile, en el mes de mayo de 1936 el gobierno del entonces presidente Alfonso López Pumarejo retoma diálogos con el profesor Joaquín Cabezas García y le ofrece el cargo de Director Nacional de Educación Física dentro del Ministerio de Educación Nacional. Este ofrecimiento es rechazado por el ilustre pedagogo chileno dada su avanzada edad, pero en cambio recomienda para que se desplace a nuestro país "una misión chilena integrada por dos técnicos en Educación Física, el profesor Candelario Sepúlveda Lafuente, inspector de Educación Física de Santiago y el profesor Ramón Quintana Carrasco, catedrático del Instituto Superior" (Gómez \& Parra, 1986, p. 37).

El profesor Candelario Sepúlveda Lafuente es nombrado Director Nacional de Educación Física y dentro de sus funciones se le encarga la organización urgente del curso de Instructores de Educación Física para maestros en comisión, que se desarrolla entre el 11 de mayo y el
5 de septiembre de 1936, con la participación de 180 alumnos, entre militares de diferentes rangos, policías, maestros en ejercicio, deportistas y jóvenes estudiantes de diferentes regiones de Colombia, que habían terminado recientemente sus estudios secundarios y que, aunque en su mayoría no conocían de Educación Física, sí decidieron formarse en esta disciplina dado el ofrecimiento que se hizo desde el gobierno de recibir una beca y la posibilidad de ubicación laboral. Del artículo primero del Decreto 1069 de mayo 12 de 1936, se puede inferir que el mencionado curso tuvo el siguiente plan de estudios: Anatomía, Fisiología, Química, Nutrición, Psicología y Gimnasia. Según Vaca (1993), el "curso fue tan intensivo, que posteriormente se validó por dos años" (p. 13) para el plan de estudios del Instituto Nacional de Educación Física. Las clases teóricas de este curso se trabajaban en las instalaciones de la Facultad de Medicina de la Universidad Nacional, ubicadas en lo que hoy en día es la dirección de reclutamiento del Ejército Nacional, en el sector de los Mártires; y la gimnasia, en la antigua Escuela Militar ubicada en lo que actualmente es el Hotel Tequendama. A su vez, el profesor Ramón Quintana Carrasco es nombrado como Asesor Técnico de la Dirección Nacional de Educación Física.

El jueves 25 de junio de 1936, mediante el Decreto 1528, expedido por el gobierno del presidente Alfonso López Pumarejo, se inicia la verdadera profesionalización del profesorado de Educación Física, al crearse el Instituto Nacional de Educación Física -mejor conocido como INEF- y asignar como su primer director al profesor Candelario Sepúlveda Lafuente, así como un presupuesto cercano a los $\$ 150.000$ pesos para el funcionamiento administrativo y académico; además, para iniciar la construcción de un edificio anexo al estadio de fútbol, en el nuevo campus de la Universidad Nacional en la ciudad universitaria. Al tenor de este decreto, al INEF se le asignó la responsabilidad de formar al profesorado, instructores y entrenadores del área que el país urgentemente estaba reclamando. En el Artículo 3 del Decreto 1528 (1936) se especifican las clases de alumnos que atenderá el Instituto Nacional de Educación Física en los siguientes términos:

Habrá dos clases de alumnos: los que siguen el curso regular, por consiguiente, el plan completo de estudios, que recibirán el título de profesores de Educación Física, y aquellos cuyas condiciones se especificarán en cada caso y que harán cursos rápidos a fin de obtener el grado de instructores 
de Educación Física, entrenadores deportivos y atléticos, masajistas y encargados de plazas de deportes y juegos infantiles (p. 1).

Figura 2. Placa conmemorativa ubicada en el Estadio de Fútbol Alfonso López de la Universidad Nacional sede Bogotá.

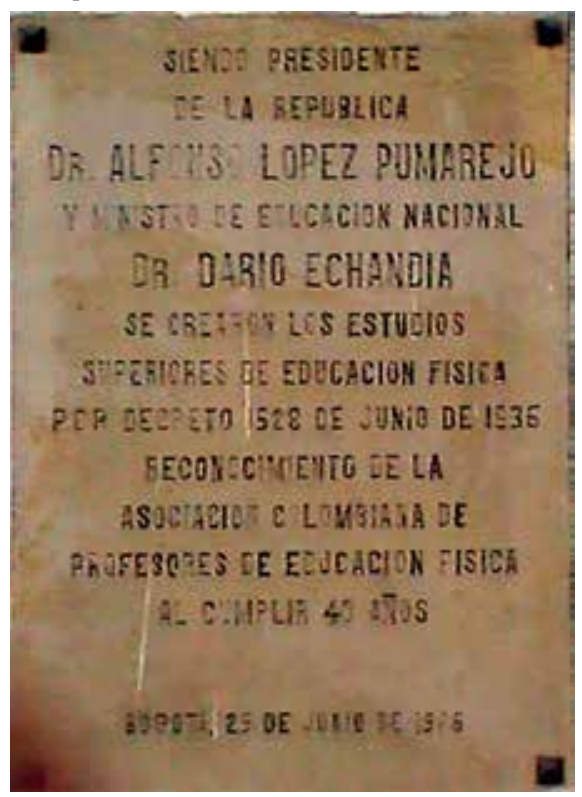

Fuente: Mg. Germán Preciado Mora.

Como respuesta a lo decretado, el 11 de septiembre de 1936, según la circular No. 45, se organizó un curso de un año de duración para la capacitación de profesores de gimnasia, el cual iniciaría el 1 de octubre y tendría las siguientes materias en su programa general (p. 1):

\footnotetext{
- Anatomía - Pedagogía - Esgrima

- Fisiología • Mecánica del Aparato • Deportes Locomotor

- Bioquímica - Gimnasia Rítmica - Gimnasia Práctica

- Biología - Primeros Auxilios Educación Física Teórica y Didáctica

- Nutrición • Música y Canto Coral

- Psicología
}

Los cursos que hasta el momento se han presentado y descrito, aunque pueden considerarse un buen intento por parte del gobierno para la capacitación del profesorado encargado de la Educación Física del país, no le quitan la génesis de la formación profesional de los educadores físicos al plan de estudios que se estableció en el artículo 15 del mismo Decreto 1528 (1936), en los siguientes términos "los estudios durarán tres años y se someterán al plan de estudios que va a continuación" (p. 3), expresados en ramos y con intensidades en horas semanales, que en el ámbito deportivo estaban divididos para hombres y para mujeres así:
Tabla 1. Plan de estudios 1936

\begin{tabular}{|c|c|c|c|}
\hline \multirow{2}{*}{ RAMOS } & \multicolumn{3}{|c|}{ AÑOS } \\
\hline & $1^{\circ}$ & $2^{o}$ & $3^{\circ}$ \\
\hline Psicología & 2 & ---- & $-\cdots$ \\
\hline Pedagogía, Historia de la Pedagogía & ---- & 2 & 2 \\
\hline Educación Teórica (Física) & ---- & 3 & 2 \\
\hline Didáctica de la Educación Física & ---- & --- & 3 \\
\hline Anatomía & 4 & ---- & ---- \\
\hline Fisiología & 2 & 2 & $\begin{array}{c}--- \\
-1\end{array}$ \\
\hline Mecánica del movimiento & $\begin{array}{ll}--- \\
-\cdots\end{array}$ & 1 & 1 \\
\hline Bioquímica & 2 & 1 & $-\cdots$ \\
\hline Biología & 2 & ---- & ---- \\
\hline Nutrición y Dietética & ---- & 1 & 1 \\
\hline Inglés (traducción) & 1 & 1 & 1 \\
\hline Primeros Auxilios & ---- & $---\cdot$ & 1 \\
\hline Puericultura (Mujeres) & ---- & ---- & 1 \\
\hline Kinesiterapia & ---- & $-\cdots$ & 2 \\
\hline Gimnasia Práctica Mujeres & 3 & 3 & 3 \\
\hline Gimnasia Práctica Hombres & 3 & 3 & 3 \\
\hline Natación Mujeres & 1 & 1 & 1 \\
\hline Natación Hombres & 1 & 1 & 1 \\
\hline Juegos Pedagógicos & 1 & 1 & ---- \\
\hline Deportes Mujeres & 1 & 1 & ---- \\
\hline Deportes Hombres & 1 & 1 & 1 \\
\hline Atletismo Mujeres & 1 & 1 & 1 \\
\hline Atletismo Hombres & 1 & 1 & 1 \\
\hline Danza y Gimnasia Rítmica Mujeres & 2 & 2 & 1 \\
\hline Gimnasia Rítmica Hombres & 1 & 1 & 1 \\
\hline Tiro al blanco Hombres & 1 & 1 & 1 \\
\hline Esgrima Mujeres & 1 & 1 & 1 \\
\hline Esgrima Hombres & 1 & 1 & 1 \\
\hline Música y canto oral & 2 & 2 & 1 \\
\hline Redacción Deportiva & ---- & --- & 1 \\
\hline Total clases semanales & -----. & ------ & $-\cdots$ \\
\hline Mujeres & 25 & 23 & 24 \\
\hline Hombres & 25 & 23 & 24 \\
\hline
\end{tabular}

Fuente: Decreto 1528 de 1936.

Teniendo ya un plan de estudios aprobado, el INEF enfoca todos sus esfuerzos en dar inicio a la formación profesional de profesores de Educación Física, por lo que se determinó el lunes 1 de febrero de 1937 como la fecha de inicio de las labores académicas para los tres cursos dispuestos para optar por el título de profesor de Educación Física. En total, sumaban 100 estudiantes de todo el territorio colombiano, algunos de los cuales se encontraban becados desde sus regiones. Por problemas en el proceso de admisión de los aspirantes al primer curso y por no haber culminado las reparaciones locativas en el local donde funcionaba el INEF dentro de la Facultad de 
Medicina de la Universidad Nacional, en el Parque de los Mártires (Carrera 15 No. 9-50), las clases se iniciaron el 15 de febrero de 1937 para los estudiantes de los cursos 2 y 3 (quienes eran los mismos que habían tomado los cursos de los que se habló anteriormente) y el 22 de febrero para los de primer año.

El transcurso del año 1937 estuvo lleno de sucesos que pusieron en riesgo la continuidad del recién iniciado proceso formativo profesional de profesores de Educación Física, entre estos se pueden mencionar:

- El regreso a Chile a inicios de febrero del Director del INEF, profesor Candelario Sepúlveda, y la designación por poco tiempo de varios directores, en su orden: Ramón Quintana Carrasco (febrero 11), Alfonso Esguerra Gómez (marzo 12), Hernando Ordoñez (julio 13).

- El cambio de plan de estudios, ya que el establecido en el artículo 15 del Decreto 1528 se modificó el 21 de agosto de 1937 mediante el Decreto 1550.

- El cambio forzoso de sede, del local dentro de la Facultad de Medicina de la Universidad Nacional a una casa arrendada ubicada en la carrera 8 No. 9-93, dada la toma, vandalismo, expulsión y lanzamiento que realizaron los estudiantes de Odontología, Medicina y Farmacia de la Universidad Nacional a las instalaciones, materiales y personal del INEF, el jueves16 de septiembre a partir de las 4 p.m.

Sin embargo, a pesar de todo lo anterior, el 21 de diciembre de 1937 se gradúan los primeros profesores de Educación Física, al culminar las asignaturas previstas para el tercer año y cumplir con todos los requisitos exigidos. El grupo de doce pioneros estuvo conformado por: Ana María Chaves Posada, Gilma Wills Olaya, Rita Perdomo, Elisa Gaviria, Rosa Cubillos, Josefina Chávez Sicard, Carlos Arias, Justo Peñalosa, Alberto Rendón, Enrique Vargas, Luis E. Sánchez y Miguel García.

De ahí en adelante y hasta nuestros días, la formación profesional de docentes para Educación Física se ha visto envuelta en una serie de acontecimientos, sucesos y decisiones, sobre todo de tipo político y normativo (generados en su mayoría por los cambios de gobierno), que en algunos momentos la han llevado a la crisis (hasta el punto de casi desaparecer al tener que sus- pender labores académicas) y en otros, han colocado al educador físico y la profesión en un pedestal muy alto dentro del sistema educativo y la sociedad colombiana, por los aportes que se han hecho tanto a la formación integral de la niñez y la juventud, como a la salud y el bienestar de la población; y a la realización de grandes movimientos, actividades y eventos: sociales, deportivos, de exhibición, culturales, académicos, investigativos y de innovación.

Figura 3. Diploma entregado a los primeros profesionales de la Educación Física colombiana.

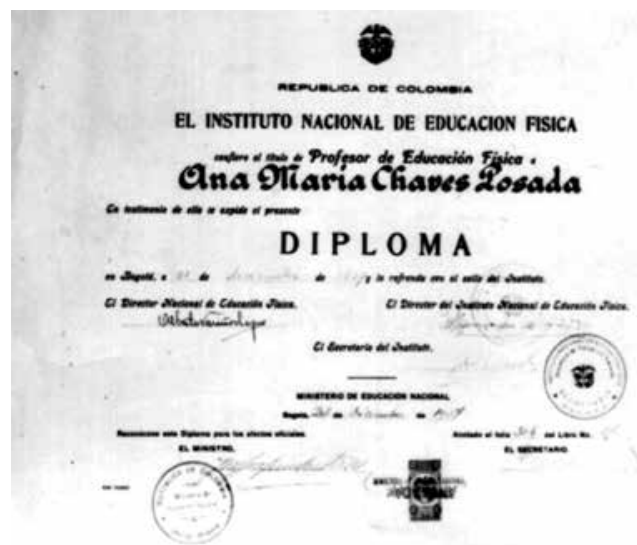

Fuente: Archivo del Grupo de Investigación Rescate Histórico de la Educación Física, el Deporte y Recreación ${ }^{2}$.

Rápidamente, se pueden mencionar los siguientes hechos históricos como hitos dentro de este recorrido de 80 años de formación profesional y de acción pedagógica de la Educación Física colombiana:

1. Entre 1937 y 1969 la formación estuvo centralizada en Bogotá y los estudiantes del resto del país debían desplazarse a la capital para poder adelantar su formación pero, gracias a que varios de estos profesionales, al regresar a sus sitios de origen, incursionaron en las Instituciones de Educación Superior de sus regiones y comenzaron a hacer ver la necesidad de la

2 El Grupo de Investigación Rescate Histórico de la Educación Física, el Deporte y Recreación estuvo conformado en la primera década del siglo veintiuno, por los docentes: Raúl Eduardo Bernal Sánchez, Mario Gildardo Loaiza Padilla, Olga Cecilia Muñoz Rojas, Germán Preciado Mora, José Olegario Rincón Velandia, Darío Ramírez Duarte(+) y su gestor, el maestro Ángel Humberto Vaca Hernández (+), y desarrolló los proyectos de investigación: Rescate histórico del saber de la Educación Física en la Universidad Pedagógica Nacional; Memoria oral y configuración de la Educación Física en Colombia entre 1936 y 1970; Fotografía y fuentes documentales escritas en la configuración de la Educación Física en Colombia entre 1936 y 1970; aprobados por el Centro de Investigaciones de la Universidad Pedagógica - CIUP para las vigencias 2004 a 2008. 
formación de Educadores Físicos y a gestionar la creación de nuevos programas -apoyados en el estudio que varias universidades hicieron y recopilaron en 1970 en el documento "Organización y ampliación de Departamentos de Educación Física en universidades colombianas"-, se inició la expansión por el territorio nacional de la siguiente forma:

- En el primer semestre del año 1969, la Universidad de Antioquia oferta e inicia la Licenciatura en Educación, área mayor: Educación Física; área menor: Salud y Recreación, adscrita a la Facultad de Educación.

- En 1971, la Universidad de Pamplona inicia en el primer semestre la formación a nivel técnico y luego, en 1974, se ofrece el programa de Licenciatura en Educación Básica con especialidad en Educación Física.

- En el segundo semestre de 1971, la Universidad Pedagógica y Tecnológica de Colombia en Tunja, UPTC, inicia su programa de Licenciatura en Educación Física.

- En 1972, la Corporación Universitaria de la Costa, Unicosta, de Barranquilla, inicia el programa de Educación Física, Recreación y Deportes, que luego se adscribe a la Universidad del Atlántico.

- En enero de 1972, la institución universitaria Unidad Central del Valle del Cauca, ubicada en Tuluá, inicia labores formativas en la Licenciatura en Educación Física.

- La Universidad del Valle, con la colaboración del convenio suscrito con el gobierno alemán, inicia la formación profesional de educadores físicos en 1975 con su programa de Licenciatura en Educación con especialidad en Educación Física y Salud.

- En el segundo semestre de 1975, el Instituto Técnico Universitario Surcolombiano, ITUSCO, hoy Universidad Surcolombiana, inicia la formación profesional con su programa de Licenciatura en Educación Física.

- En febrero de 1977, el Instituto Técnico Universitario de Cundinamarca, ITUC, hoy Universidad de Cundinamarca, da apertura al nuevo programa de Licenciatura en Ciencias de la Educación con Especialidad en Educación Física en su sede de Fusagasugá.
- En enero de 1995, la Universidad del Tolima en Ibagué inicia las labores formativas de educadores físicos con su programa de Licenciatura en Educación Física, Deportes y Recreación.

De esta manera, se continúa la creación de programas por todo el territorio colombiano hasta llegar a la actualidad, año 2016, con una oferta de 84 programas de formación y presencia en 36 municipios y ciudades de Colombia, en 48 Instituciones de Educación Superior, lo que facilita y hace posible la elección y el acceso de la juventud colombiana a la Educación Física como proyecto de vida profesional.

2. Dentro de las Instituciones que han sido responsables de la formación, podemos mencionar:

- El Instituto Nacional de Educación Física, INEF. Dependiente del Ministerio de Educación Nacional de 1937 a 1939, de la Universidad Nacional de 1939 a 1942 y de la Escuela Normal Superior Universitaria de 1942 a 1951.

- La Escuela Nacional de Educación Física, ENEF. Dependiente del Ministerio de Educación Nacional de 1952 a 1954 funcionaba como una sola institución, pero con ramas de mujeres y hombres separadas. Luego, de 1954 a 1959, continuó dentro del MEN pero como dos instituciones independientes: Escuela Nacional de Educación Física para Varones y Escuela Nacional de Educación Física para Señoritas, situación que se remedió unificándola en 1959; funcionó de esta forma hasta el 2 de agosto de 1962 cuando se incorporó a la UPN.

- La Universidad Pedagógica Nacional. Como Facultad de Educación Física y Salud de 1962 a 1969, como Departamento de Educación Física de 1969 a 1994 y de ahí en adelante hasta hoy, 2016, nuevamente como Facultad de Educación Física.

Los cambios en las entidades responsables de la formación de profesores para la Educación Física que se acaban de mostrar produjeron nueve mudanzas de sedes y locales dentro de la geografía bogotana de la siguiente forma:

- Se inician labores en la Facultad de Medicina de la Universidad Nacional en el Parque de los Mártires (Carrera 15 No. 9-50), hasta el 16 de septiembre de 1937, fecha del intempestivo desalojo por 
parte de los estudiantes de la Universidad Nacional del que ya hablamos y que provocó el cambio forzoso a la nueva sede.

- El 22 de septiembre de 1937 se llega a una casa arrendada, ubicada en la carrera 8 No. 9-93, en el marco de la plaza de Bolívar.

- A principios del año 1939 se traslada a la tercera sede, en la Ciudad Universitaria, al edificio especialmente planeado y construido para la Educación Física, ubicado rodeando la parte trasera de la tribuna de eventos del estadio Alfonso López Pumarejo.

- El 27 de enero de 1942 llega la Educación Física a su cuarta morada en la calle 13 No. 16-74 (aledaña a la estación de la Sabana) adjunta a la Escuela Normal Superior.

- El 13 de mayo de 1952 se establece en la parte alta del Parque Nacional (hoy instalaciones de la policía de Carabineros) la Escuela Nacional de Educación Física para varones, mientras que la rama femenina se había establecido en una casa vecina a la Universidad Pedagógica Femenina, calle 73 No. 13-30, donde funcionó por algún tiempo, para pasar luego a otra casa en la calle 74A No. 4-23.

- Cuando se unificaron las dos secciones se continuó trabajando en el Parque Nacional, un tiempo en la parte alta y luego en lo que en la actualidad es la sede de artes de la UPN, en el coliseo del Parque Nacional, lugar que correspondería a la sexta sede para la Educación Física.

- A mediados del año 1967 se debe arrendar una casa grande en la avenida 39 No. 7-84 para trasladar allí las oficinas administrativas y algunas aulas, constituyendo esta la séptima sede.

- A comienzos de 1969, por fin se llega a las instalaciones de la avenida Chile de la Universidad Pedagógica Nacional, al edificio abandonado del costado sur occidental donde había funcionado el Instituto Pedagógico Nacional en la calle 72, el cual estaba desocupado por el deterioro sufrido a causa de un fuerte temblor que había afectado a la capital colombiana. Allí se instalan en salones mal acondicionados, las oficinas de la decanatura y el depósito, mientras las aulas se localizan en los edificios A y B de la Universidad. Esta sería la octava sede.

Figura 4. Mosaico de fotografías de algunas de las sedes de la Educación Física en Bogotá.

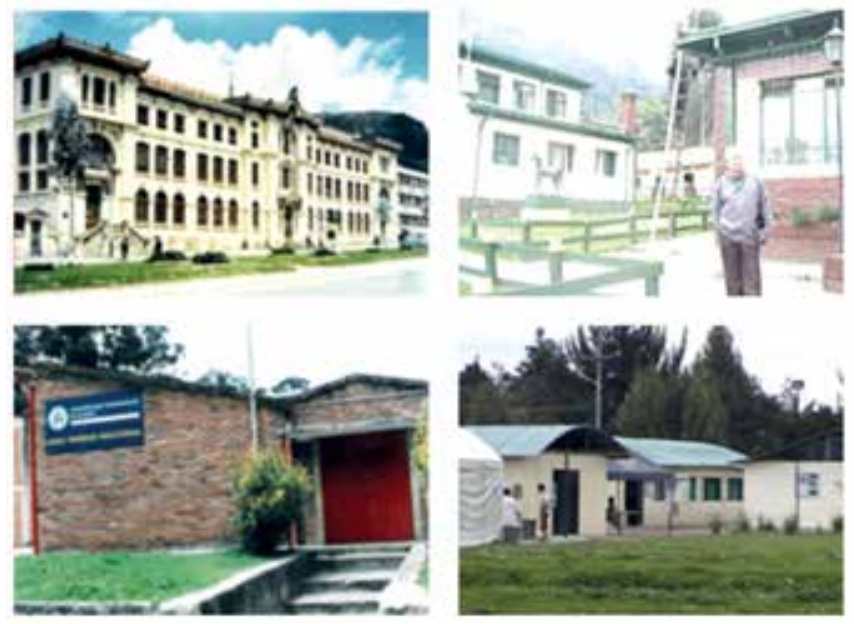

Fuente: Archivo del Grupo de Investigación Rescate Histórico de la Educación Física, el Deporte y Recreación.

- El 21 de febrero de 2007 se emprende el viaje a la novena y actual sede de la Facultad de Educación Física de la UPN, la sede Valmaría, ubicada en un lote de 31.3 hectáreas, en la calle 183 con carrera 54D, vecino del Centro Comercial Santafé y de los talleres del norte de Transmilenio; una sede que promete, y esperamos que así sea, ser pensada y construida para las necesidades actuales de la Facultad de Educación Física de la Universidad.

3. A lo largo de los 80 años de formación profesional, los programas del país se han visto apoyados por varias misiones extranjeras, contratadas por los gobiernos de turno para impulsar y consolidar dicha formación. Entre ellas cabe destacar: la chilena (1936-1937), tres alemanas (1924 a 1927, 1953 a 1957 y 1965 a 1984), una española (1952), tres estadounidenses con la Alianza para el Progreso (1961 a 1975), los Cuerpos de Paz y la Misión Fulbright. Asimismo, se han suscrito con el mismo fin varios Convenios de cooperación con países como: México, antigua Unión Soviética, Cuba, Francia y Argentina. Además, muchos de los docentes de los programas de todo el país han viajado a diferentes universidades del planeta a adelantar programas de formación posgradual de especializaciones, maestrías y doctorados. 
4. También ha evolucionado en estos 80 años el tiempo requerido para la formación de los profesionales, el cual ha pasado de tres años (1936 a 1961), a cuatro años (1962 a 1968); cuatro años repartidos en 8 semestres (1969 a 1985) y 9 semestres (de 1986 a 1998, en la UPN); y hoy en día, a cinco años para estudios diurnos y seis para nocturnos, distribuidos en 10 y 12 semestres, respectivamente, para todas las 48 Instituciones de Educación Superior del país.

5. Las titulaciones que se han otorgado han sido de: Profesor de Educación Física (1936 a 1941); Licenciado en Ciencias de la Educación Especializado en Educación Física (1942 a 1950); Licenciado en Educación Física (1951 a 1964); Licenciado con Estudios en Educación Física (1965 a 1986). A partir de la expansión por las diferentes regiones de la geografía colombiana, aparecen nuevas titulaciones entre las cuales podemos mencionar: Licenciado en Educación Física y Deportes; Licenciado en Educación Física, Recreación y Deportes; Licenciado en Deporte; Licenciado en Recreación; Licenciado en Recreación y Turismo; Licenciado en Cultura Física, Recreación y Deportes; Licenciado en Educación Básica con énfasis en Educación Física, Recreación y Deportes; Profesional en Ciencias del Deporte; Profesional en Ciencias del Deporte y la Recreación; Profesional en Ciencias del Deporte y la Actividad Física; Profesional en Cultura Física, Deporte y Recreación; Profesional en Cultura Física y Deporte; Profesional en Ciencias del Deporte y la Educación Física; Profesional en Actividad Física y Deporte; Profesional en Deporte; Profesional en Recreación; Profesional en Deporte; Profesional en Deporte y Actividad Física; Profesional en Deporte y Cultura Física; Profesional en Actividad Física y Deporte; Profesional en Entrenamiento Deportivo; Profesional en Entrenamiento Deportivo y Preparación Física, Administrador Deportivo.

\section{EL PRESENTE DE LA FORMACIÓN DE DOCENTES PARA LA EDUCACIÓN FÍSICA COLOMBIANA}

En este año 2016, y después de ochenta años de acción pedagógica profesional de la Educación Física, las posibilidades de formación que encontramos para maestros del área muestran una amplia gama de programas para todos los niveles de nuestro actual sistema de Educa- ción superior. La fotografía del Sistema de Formación Profesional de maestros que pueden ejercer como docentes del área fundamental de Educación Física, Recreación y Deportes (Ley 115 de 1994) en entidades escolares públicas o privadas de Colombia nos muestra lo siguiente:

- Según el Estatuto de Profesionalización Docente (Decreto 1278 de junio 19 de 2002), pueden ejercer la función de docentes: los normalistas superiores (solo pueden laborar en el nivel de Educación preescolar y básica primaria), los profesionales de la Educación (Licenciados) y los profesionales de otras áreas del conocimiento, con título diferente al de licenciado (que demuestren haber adelantado curso de pedagogía de 480 horas o posgrado en educación). Los licenciados y los demás profesionales mencionados, pueden desempeñarse en los niveles de Educación preescolar, básica (primaria y secundaria) y media, según la especialidad de formación.

Figura 5. Clase de Educación Física Mental en el Colegio Distrital Rafael Uribe Uribe.

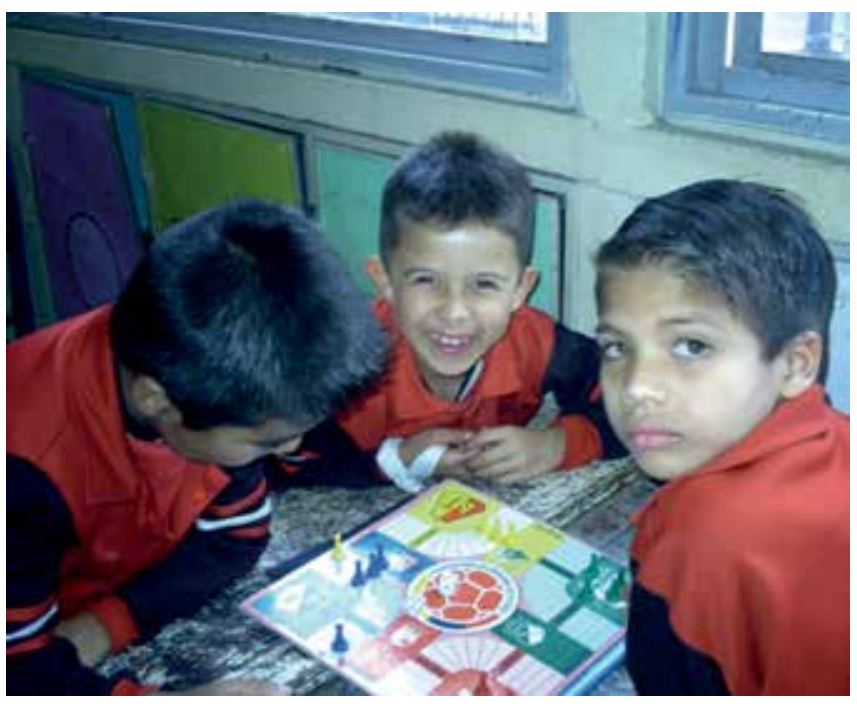

Fuente: Mg. Germán Preciado Mora.

- Las instituciones encargadas de la formación inicial de maestros están compuestas por: las Escuelas Normales Superiores quienes otorgan el título de Normalista Superior y las Facultades de Educación quienes otorgan el título de Licenciados en las áreas básicas o certificado de curso de pedagogía para profesionales no licenciados. 
- Las Facultades de Educación, a su vez, ofrecen dos niveles de formación: uno de pregrado (para formación profesional inicial) y otro de posgrado (de formación avanzada para profesionales graduados). En pregrado se ofertan programas de carácter Técnico Profesional, Tecnológico y Profesional. En posgrado se ofrecen Especializaciones, Maestrías y Doctorados. Cabe aclarar que en los últimos años el Servicio Nacional de Aprendizaje, SENA, también ha entrado en el mercado ofreciendo programas de nivel Técnico Profesional y Tecnológico.

- En términos de la duración, la formación complementaria en las Escuelas Normales Superiores es de dos años (en el caso de los bachilleres con profundización en pedagogía) y de dos años y medio en los demás casos. La licenciatura es de cinco años, en los programas diurnos y de 6 años, en los programas nocturnos. Los dos tipos de formación tienen como requisito de ingreso el título de bachiller, bien sea, académico, técnico o con profundización en pedagogía.

- En relación con la presencialidad, encontramos dos posibilidades: presencial y a distancia.

\section{ESCUELAS NORMALES SUPERIORES ${ }^{3}$}

Actualmente, el país cuenta con 137 Escuelas Normales Superiores -ENS-, debidamente autorizadas por el Ministerio de Educación Nacional, que ofertan igual número de programas de formación complementaria en metodología presencial. Encontramos 130 de carácter oficial y 7 privadas, distribuidas por todo el territorio nacional así: Amazonas 1, Antioquia 23, Arauca 1, Atlántico 7, Bolívar 3, Boyacá 10, Caldas 10, Caquetá 1, Casanare 1, Cauca 5, Cesar 2, Chocó 7, Córdoba 3, Cundinamarca 11, Huila 3, Guajira 2, Magdalena 2, Meta 3, Nariño 6, Norte de Santander 4, Putumayo 1, Quindío 1, Risaralda 1, Santander 10, Sucre 3, Tolima 4, Valle del Cauca 7, Vaupés 1, Vichada 1, Bogotá 3.

3 Datos y cifras tomadas de la Asociación Nacional de Escuelas Normales Superiores ASONEN http://www.asonen.org.co/integrantes_por_regiones.html
Estas instituciones se han agrupado en la Asociación Nacional de Escuelas Normales Superiores, ASONEN, y han establecido convenios con las Facultades de Educación para que sus egresados puedan continuar su formación como licenciados.

\section{FACULTADES DE EDUCACIÓN ${ }^{4}$}

Actualmente, Colombia cuenta con 48 Instituciones de Educación Superior (distribuidas por la geografía nacional en 36 municipios y ciudades) debidamente autorizadas por el Estado, para ofertar un total de 84 programas de formación profesional en el campo de la Educación Física, Deporte, Recreación y campos disciplinares o de prácticas afines. Estos programas se distribuyen en los niveles de formación de la Educación Superior así: para el nivel Técnico Profesional, 9 programas; para el nivel Tecnológico, 18 programas, y para Profesional, 57 programas. En cuanto a la presencialidad, se cuenta con 79 programas con metodología presencial y 5 a distancia.

Figura 6. Clase de Campismo para docentes en formación en la Universidad Libre de Colombia sede Bosque Popular.

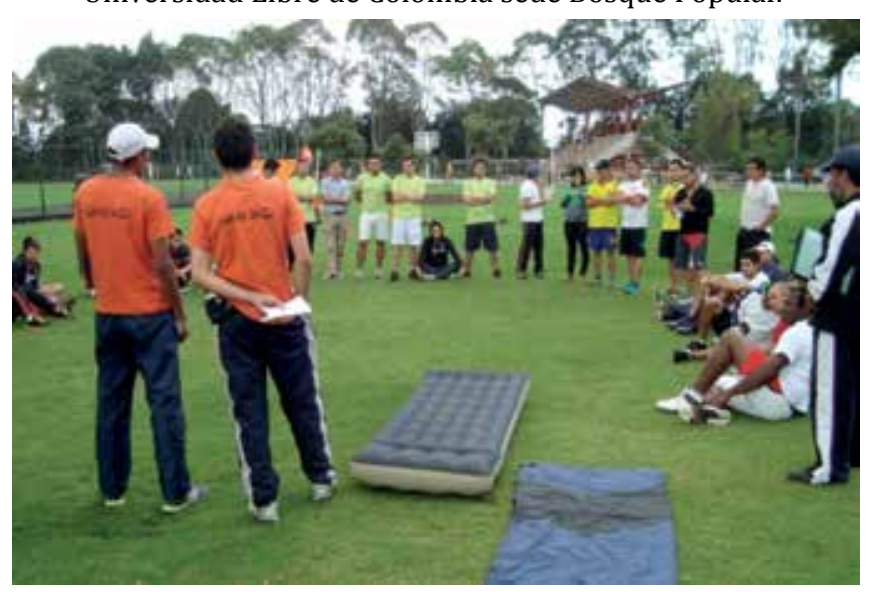

Fuente: Mg. Germán Preciado Mora.

En la tabla 2, se observa el consolidado al año 2016 de Instituciones de Educación Superior, su localización regional y los programas de formación en los niveles de Técnico Profesional, Tecnológico y Profesional ofertados, que cuentan con el reconocimiento del Ministerio de Educación Nacional.

4 Datos y cifras procesadas a partir de la información del Sistema Nacional de Información de la Educación Superior SNIES - http://snies.mineducacion.gov.co/consultasnies/programa y de la Asociación Red Colombiana de Facultades de Deporte, Educación Física y Recreación ARCOFADER http:// www.arcofader.org/http sitesgooglecomsitesisinfoarcofader 
Tabla 2. Consolidado al año 2016 de Instituciones de Educación Superior que cuentan con el reconocimiento del Ministerio de Educación Nacional.

\begin{tabular}{|c|c|c|c|c|c|}
\hline \multirow{2}{*}{ No. } & \multirow{2}{*}{ Institución De Educación Superior } & \multirow{2}{*}{ Localización } & \multicolumn{3}{|c|}{ Nivel de Formación y Nombre del Programa } \\
\hline & & & Técnico Profesional & Tecnológico & Profesional \\
\hline 1 & Corporación De Estudios Superiores Saligmandra & Cali & $\begin{array}{l}\text { Técnica profesional en } \\
\text { Educación Fisica y } \\
\text { Recreación } \\
\text { Técnica profesional en } \\
\text { Prevención y } \\
\text { Rehabiltación a través } \\
\text { del Deporte }\end{array}$ & & \\
\hline 2 & Corporación John F Kennedy & Bogoti. D.C. & & $\begin{array}{l}\text { Teconologis en Deporte y } \\
\text { Recresción }\end{array}$ & \\
\hline 3 & Corporacón Universtaria Autónoma del Cauca & Popayán & & & Deporte y Actividad Fisica \\
\hline 4 & Corporación Universitaria CENDA & Bogotá. D.C. & $\begin{array}{l}\text { Técrica profesional en } \\
\text { Educacón Fisica } \\
\text { Técnica profesional en } \\
\text { Entrenamiento Deportivo }\end{array}$ & & \\
\hline 5 & $\begin{array}{c}\text { Corporación Universtaria De Investigación Y } \\
\text { Desarrolo. UDI }\end{array}$ & Bucaramangs & & & $\begin{array}{l}\text { Entrenamiento Deportivo. } \\
\text { Preparación Fisica }\end{array}$ \\
\hline 6 & Corporación Universitarig Del Caribe - CECAR & Sincelejo & & & $\begin{array}{l}\text { Ciencias Del Deporte y la } \\
\text { Actividad Fisica }\end{array}$ \\
\hline 7 & Corporación Universtaria Latinosmenicana - CUL & Bamanquilig & & & $\begin{array}{c}\text { Licenciatura en } \\
\text { Educación Básica con } \\
\text { Enfasis en Educación } \\
\text { Fisica, Recreación y } \\
\text { Deportes }\end{array}$ \\
\hline 8 & $\begin{array}{c}\text { Corporacón Universtaria Mnuto De Dios. } \\
\text { UNIMINUTOO }\end{array}$ & Bogotá, D.C. & & & $\begin{array}{c}\text { Licencistura en } \\
\text { Educación Fisica } \\
\text { Recreación y Deporte }\end{array}$ \\
\hline 9 & $\begin{array}{c}\text { Esouela Mitar De Cadetes 'General José Maria } \\
\text { Córdova' }\end{array}$ & Bogota. D.C. & & & Educación Fisica Mitar \\
\hline 10 & $\begin{array}{l}\text { Fundación De Educación Superior San José } \\
\text { FESSANOSE }\end{array}$ & Bogots. D.C. & $\begin{array}{l}\text { Técrica profesional en } \\
\text { Recreación Dingida } \\
\text { Téconica profesional en } \\
\text { Rendimiento Deportivo }\end{array}$ & $\begin{array}{l}\text { Teconologis en } \\
\text { Rendimiento Deportivo }\end{array}$ & \\
\hline 11 & Fundación Tecnológica Autónoma De Bogotá FABA & Bogota, D.C. & & $\begin{array}{c}\text { Tecnologís en } \\
\text { Recreación Dirigida }\end{array}$ & \\
\hline 12 & Fundación Universtaris Del Ares Andins & Bogotá. D.C. & $\begin{array}{l}\text { Técnico profesional en } \\
\text { Entrengmiento Deportivo }\end{array}$ & $\begin{array}{c}\text { Tecnologia Deportiva } \\
\text { Tecnologia Deportiva } \\
\text { para Personas con } \\
\text { Discapscidsd } \\
\text { Teconologis en } \\
\text { Entrengmiento del Fútbol }\end{array}$ & \\
\hline 13 & Fundación Universtaria Juan De Castelanos & Tunja & & & $\begin{array}{c}\text { Licencisturs en } \\
\text { Educación Fisica } \\
\text { Recreación y Deportes }\end{array}$ \\
\hline 14 & Fundacón Universtaria Luis Amigo FUNLAM & Medelin & & & Actividad Fisica y Deporte \\
\hline 15 & $\begin{array}{l}\text { Institudion Universataris Centro De Estudios } \\
\text { Superiores Maris Goretti CESMAG }\end{array}$ & Pasto & & & $\begin{array}{l}\text { Lidencistura en } \\
\text { Educsción Física }\end{array}$ \\
\hline 16 & $\begin{array}{l}\text { Institución Universtaria } \\
\text { Escuels Nacional Del Deporte }\end{array}$ & Cali & & $\begin{array}{l}\text { Teconología Deportiva } \\
\text { Tecnologia en Evaluacón } \\
\text { y Programacoión de la } \\
\text { Actividad Fisica }\end{array}$ & Deporte \\
\hline 17 & $\begin{array}{l}\text { Institución Universtaria Poiliéconico } \\
\text { Jaime Isaza Cadavid }\end{array}$ & Medellin & & $\begin{array}{l}\text { Tecnologis Deportiva } \\
\text { (Presencial y Distancia) } \\
\text { Teconología en Educación } \\
\text { Fisica - Teconologis en } \\
\text { Entrensmiento Deportivo } \\
\text { Teconologis en } \\
\text { Recreación Dirgids }\end{array}$ & $\begin{array}{l}\text { Lioenciatura en } \\
\text { Educación Básica con } \\
\text { Enfasis en Educación } \\
\text { Fisica, Recreación y } \\
\text { Deporte } \\
\text { Profesiongl en Deporte }\end{array}$ \\
\hline 18 & Instituto Tecnológico Del Putumayo & Mocos & & $\begin{array}{l}\text { Tecnología en Educrabón } \\
\text { Fisics }\end{array}$ & \\
\hline 19 & Unidad Central Del Vale Del Cauca & Tuluá & & & $\begin{array}{l}\text { Licenciatura en } \\
\text { Educación Básica con } \\
\text { Enfasis en Educación } \\
\text { Fisica, Recreación y } \\
\text { Deportes }\end{array}$ \\
\hline 20 & Unidades Tecnológicas De Santander & $\begin{array}{l}\text { Cúcuts } \\
\text { Bucaramangs }\end{array}$ & & Tecnologia Deportiva & $\begin{array}{l}\text { Protesional en Actividad } \\
\text { Fisicg y Deporte } \\
\text { (En } 2 \text { Municipios) }\end{array}$ \\
\hline 21 & Universidad Autonoma DelCanbe & Barranquilia & & & Deporte y Cutura Fisica \\
\hline 22 & Universidad Católica De Onente & Rionegro & & & $\begin{array}{c}\text { Licenciatura en } \\
\text { Educsción Fisica } \\
\text { Recreación y Deportes }\end{array}$ \\
\hline 23 & Universidad Cooperativa De Colombis & Bucaramanga & $\begin{array}{l}\text { Técnica profesional en } \\
\text { Fútbol }\end{array}$ & & $\begin{array}{c}\text { Licenciatura en } \\
\text { Educación Fisica } \\
\text { Recreación y Deporte }\end{array}$ \\
\hline 24 & Universidad De Antioquia & $\begin{array}{l}\text { Medellin, Amaifi, } \\
\text { Andes, Carepg. } \\
\text { Carmen de Viboral, } \\
\text { Puerto Berno, Turbo }\end{array}$ & & & $\begin{array}{l}\text { Licenciatura en } \\
\text { Educación Fisica } \\
\text { (En } 6 \text { Municipios) } \\
\text { Entrenamiento Deportivo } \\
\text { (En } 2 \text { Municipios) }\end{array}$ \\
\hline 25 & Universidad De Caldas & Manizales & & & $\begin{array}{l}\text { Lic. en Educacón Básics } \\
\text { con Ennfasisen Educaoón } \\
\text { Fisicas. Recreación y } \\
\text { Depontes }\end{array}$ \\
\hline
\end{tabular}




\begin{tabular}{|c|c|c|c|c|c|}
\hline \multirow{2}{*}{ No. } & \multirow{2}{*}{ Institución De Educación Superior } & \multirow{2}{*}{ Localización } & \multicolumn{3}{|c|}{ Nivel de Formación y Nombre del Programa } \\
\hline & & & Técnico Profesional & Tecnológico & Profesional \\
\hline 26 & $\begin{array}{l}\text { Universidad De Clencias Aplicadas Y Ambientales } \\
\text { UDCA }\end{array}$ & Bogotá. D.C. & $\begin{array}{l}\text { Técnico profesional en } \\
\text { Entrenamiento Deportivo }\end{array}$ & & Ciencias Del Deporte \\
\hline 27 & Universidad De Córdoba & Monteris & & & $\begin{array}{c}\text { Licenciatura en } \\
\text { Educación Fisica } \\
\text { Recreación y Deportes }\end{array}$ \\
\hline 28 & $\begin{array}{l}\text { Universidad De Cundinamarca } \\
\text { UDEC }\end{array}$ & $\begin{array}{l}\text { Fussgasugá } \\
\text { Sogchs }\end{array}$ & & & $\begin{array}{c}\text { Licenciatura en } \\
\text { Educación Básica con } \\
\text { Ėnfasis en Educación } \\
\text { Fisica, Recreación y } \\
\text { Deportes } \\
\text { Profesionsl en Clencias } \\
\text { del Deporte y la } \\
\text { Educación Fisica }\end{array}$ \\
\hline 29 & Universidgd De lbagué & lbagué & & $\begin{array}{c}\text { Tecnologís en } \\
\text { Entrensmiento Deportivo } \\
\text { en Fútbol }\end{array}$ & \\
\hline 30 & Universidgd De La Gugjra & Riohschs & & & $\begin{array}{c}\text { Lioencigtura en } \\
\text { Educación Fisica } \\
\text { Recreación y Deporte }\end{array}$ \\
\hline 31 & Universidad De Los Llanos & Vllavicencio & & & $\begin{array}{c}\text { Lioenciatura en } \\
\text { Educación Fisica y } \\
\text { Deportes }\end{array}$ \\
\hline 32 & Universidad De Pamplona & Pamplona & & & $\begin{array}{c}\text { Licenciatura en } \\
\text { Educación Básica con } \\
\text { Enfasis en Educación } \\
\text { Fisica, Recreación y } \\
\text { Deportes }\end{array}$ \\
\hline 33 & Universidad De San Buenaventura & $\begin{array}{c}\text { Medellin } \\
\text { Cartagens }\end{array}$ & & & $\begin{array}{c}\text { Licenciatura en } \\
\text { Educación Fisica y } \\
\text { Deportes } \\
\text { Lioenciatura en } \\
\text { Educación Fisica } \\
\text { Recreación y Deportes }\end{array}$ \\
\hline 34 & Universidad Del Atlántico & Barranquilla & & & $\begin{array}{l}\text { Licenciaturs en Cultura } \\
\text { Fisics, Recreación y } \\
\text { Deportes }\end{array}$ \\
\hline 35 & Universidad Del Cauca & Popsyán & & & $\begin{array}{c}\text { Licenciatura en } \\
\text { Educación Básica con } \\
\text { Enfasis en Educación } \\
\text { Fisica, Recreación y } \\
\text { Deporte }\end{array}$ \\
\hline
\end{tabular}

Fuente: elaboración propia.

Nota: se incluyen localización regional y los programas de formación ofertados en los niveles de Técnico Profesional, Tecnológico y Profesional.

Para finalizar, se presentan algunas de las posibilidades de desempeño laboral que tienen hoy año 2016, los profesionales que se forman en el campo de la Educación Física, Deporte, Recreación y campos disciplinares o de prácticas afines:

- Docente en educación formal niveles preescolar, básica, media, técnica, tecnológica y universitaria en instituciones públicas y privadas. Atendiendo la formación de todas las poblaciones (con y sin limitaciones y con capacidades excepcionales).

- Instructor de actividad física.

- Docentey/o Entrenador en escuelas de formación.

- Entrenador personal.

- Entrenador deportivo en centros de alto rendimiento para deportistas y/o equipos deportivos.

- Preparador físico de grupos y/o equipos deportivos.

- Instructor en gimnasios.

- Recreador y/o Animador sociocultural comunitario.

- Coordinador de programas para el tiempo libre.
- Monitor de deportes.

- Dirigente deportivo de clubes, ligas, federaciones y Comités Olímpicos Nacionales (CON).

- Supervisor en diferentes disciplinas deportivas.

- Gestor deportivo de asociaciones deportivas.

- Gestor de actividades deportivas.

- Director de procesos administrativos o formativos en empresas dedicadas al ofrecimiento de programas para la cultura física, ya sean de carácter oficial, privado y/u ONG.

- Emprendedor creando empresas de uniformes, materiales e implementos, de animación y recreación, asesorías deportivas, colegios de árbitros, servicios turísticos.

- Director o Instructor en centros de recuperación y/o rehabilitación física.

- Docente en establecimientos de educación no formal y comunidades, liderando y desarrollando procesos de formación educativa, deportiva, recreacional, cultural, artística y de salud, en el ámbito comunitario, laboral, técnico, ambiental o cívico. 
- Dirigente en organismos del sistema nacional del deporte, en universidades, clubes y/o empresas públicas y privadas.

\section{A MANERA DE CONCLUSIÓN}

Durante estos 80 años de acción pedagógica profesional de la Educación Física colombiana que estamos celebrando, se han vivido una serie de crisis y triunfos en el desarrollo, expansión y consolidación de esta profesión dentro de la sociedad colombiana, la cual ha encontrado en ella el mejor aliado para el mejoramiento de la salud y el acercamiento al bienestar general de la población y al desarrollo de sus proyectos de vida.

En este mismo periodo, la Educación Física ha permitido que muchos profesionales hombres y mujeres colombianos puedan delinear y desarrollar a su alrededor, verdaderos proyectos de vida personal y profesional; ayudando, de paso, con su ardua e infatigable labor, a que los niños, niñas, jóvenes, adultos y mayores de nuestro país tengan la orientación profesional que necesitan para sus requerimientos y necesidades de práctica, aprendizaje y/o competencia, de actividades de educación física, deporte, recreación y aprovechamiento adecuado del tiempo libre.

Después de 80 años de presencia profesional en Colombia, el panorama actual en cuanto a la formación de docentes para el campo de la Educación Física, Deporte, Recreación y disciplinas o prácticas afines, muestra un total de 48 Instituciones de Educación Superior, distribuidas en 36 municipios y ciudades, las cuales ofertan un total de 84 programas que cuentan con la aprobación del MEN.

\section{REFERENCIAS BIBLIOGRÁFICAS}

Asociación Nacional de Escuelas Normales Superiores, ASONEN (s. f.). http://www.asonen.org.co/integrantes _por_regiones.html.

Asociación Red Colombiana de Facultades de Deporte, Educación Física y Recreación, ARCOFADER (s. f.). http://www.arcofader.org/httpsitesgooglecomsi tesisin foarcofader.

Gómez, A. \& Parra, L. (1986). 50 Años de Oro - Historia de la Educación Física en Colombia como Profesión 1936 1986. Bogotá, D. E.: Publicaciones Universidad Central.

Grupo de investigación Rescate histórico de la Educación Física, el deporte y la recreación. Archivos documentales y fotográficos.

Ministerio de Educación Nacional (s. f.). Sistema Nacional de Información de la Educación Superior SNIES. Disponible en: http://snies.mineducacion.gov.co/ consultasnies/programa.

Vaca, Á. (1993). Historia del Alma Mater de la Educación Física Colombiana. Primera Parte. Bogotá, D.C.: Publicaciones Universidad Pedagógica Nacional.

Vaca, Á. (2000). Historia del Alma Mater de la Educación Física Colombiana. Segunda Parte. Bogotá, D.C.: Publicaciones Universidad Pedagógica Nacional. 